\title{
Are Mobile Applications Effective on Preventing Urinary Incontinence and Reducing Urinary Incontinence Symptoms?: A Review
}

\author{
Özge BUDAYCI, Pınar SERÇEKUŞ, Sevgi ÖZKAN \\ Pamukkale University, Faculty of Health Sciences, Denizli, TURKEY \\ Email: pinarsercekus@gmail.com
}

Type: Research Article (Received: 10.02.2020 - Corrected: ---- ～- Accepted: 26.03.2020)

\begin{abstract}
Urinary incontinence (UI) is a condition which frequently occurs among the adult women and emerges as a typical symptom of pelvic floor disorders. Kegel exercise is an effective method for the treatment of urinary incontinence by strengthening the pelvic floor muscles. On the other hand, failure to maintain continuity with exercise is a major problem. New approaches are needed to solve this problem. Today, with the increasing use of mobile technology, the use of mobile health applications has increased. Mobile applications which are being more popular can be an effective treatment option for teaching these exercises to the patients. There are many mobile applications developed in this field, but there is not enough study on the effects of these mobile applications on health. The purpose of this paper is to evaluate the effects of mobile applications involving pelvic floor exercise on the prevention and treatment of urinary incontinence by examining evidence-based research. Recent evidence-based studies have shown that mobile applications of urinary incontinence reduce symptoms, make pelvic floor exercise continue, improve quality of life, speed up healing process, increase selfmanagement and effectiveness in treating patients, and provide convenience access to care. These results suggest that the use of mobile apps of Kegel exercise may be recommended to the patients suffering from urinary incontinence.
\end{abstract}

Keywords: Kegel Exercise, Mobile Application, Pelvic Floor Muscle Training, Urinary Incontinence 


\section{Introduction}

Urinary incontinence (UI) is a condition which frequently occurs among the adult women and emerges as a typical symptom of pelvic floor disorders (Bo et al., 2017). In a review to determine UI prevalence, it was concluded that the prevalence of UI ranges from 5\% to $70 \%$. Prevalence rates increase with getting older and more than $40 \%$ of the female population is affected in women those who are older than 70 years-old (Milsom \& Gyhagen, 2019). In a study conducted in our country, it was found that the prevalence of urinary incontinence was \%14.6 (Çayan et al., 2016).

In their systematic review by Radzimińska et al. (2018) was demonstrated that pelvic floor exercise is an effective treatment for urinary incontinence in women. Pelvic floor exercise is an effective treatment option for women with stress urinary incontinence used without the need for surgical treatment. It can also be recommended as a first-line preventive treatment for older women. Pelvic floor exercise should be performed for at least 6 weeks to be effective; therefore, continuity of treatment is essential to success (Radzimińska et al., 2018). On the other hand, lack of continuity of exercise is an important problem. There are differences between continuity of short-term treatment (kegel exercise during incontinence treatment in the health care facility) and continuity of long-term treatment (self-kegel exercise after completion of treatment). Clinicians estimate that only $23 \%$ of patients keep to treatment for a long time. The most common problems in providing continuity in treatment are the difficulty of remembering the exercise and lacking of time (Borello-France et al., 2010).

In "Digital in 2019" internet, mobile phone and social media usage statistics report, there are 5.11 billion mobile phone users in the world and this constitutes $67 \%$ of the world population. $77 \%$ of mobile phone users are smartphone users ("DIGITAL 2019: GLOBAL INTERNET USE ACCELERATES,” 2019). The use of mobile health has positive characteristics such as increasing commitment to treatment and also reducing health expenditures (Hamine, Gerthguyette, Faulx, Green, \& Sarah, 2015). Mobile applications which are being more popular can be an effective treatment option for teaching these exercises to the patients. (Han, Grisales, \& Sridhar, 2017). There are many mobile applications developed in this subject. These mobile applications vary by many parameters. (Latorre, de Fraga, Seleme, Mueller, \& Berghmans, 2019). Although eHealth is a promising and developing technology, urogynecology applications have not been adequately tested or differences between usual care and those applications have not been investigated. In addition, there are not enough studies related to the utiliser's experiences and preferences (Loohuis et al., 2018).

In a study compared adherence in the treatment of Kegel exercise, mobile applications were shown to be more effective than by using written material (Araujo, Marques, \& Juliato, 2019). More than half of the women who use a mobile application that includes Kegel exercise think that mobile applications are an effective and successful treatment option in the treatment of urinary incontinence. At the same time, its providing weight control, increasing adherence to the treatment and making the patients gain self-management were defined as the factors affecting the success of mobile applications (Nyström, Asklund, Sjöström, Stenlund, \& Samuelsson, 2017). It was shown in the studies that mobile applications reduce the severity of symptoms and therefore they increase the women's life qualities (Asklund et al., 2016; Hoffman, Söderström, \& Samuelsson, 2017). Because of all these reasons, mobile applications for kegel exercise can be among the alternative treatment methods that can be used in clinical practice (Saboia et al., 2019). Pregnancy and giving a birth are known as important risk factors causing the perineum and pelvic floor weaken and get injured (Leroy, Lúcio, \& Lopes, 2016). For this reason, mobile applications can be used as a supportive 
therapy not only for elderly women, but also for women who have postpartum urinary incontinence problems (Saboia et al., 2019). Studies on the health effects of mobile applications is not enough. There is a need for more researches regards to effects of these practices on health. The purpose of this review is to evaluate the effects of mobile applications including pelvic floor exercise in the prevention and treatment of urinary incontinence.

\section{The Effect of Mobile Applications for Pelvic Floor Exercise on Urinary Incontinence}

There are few evidence-based studies examining the effect of mobile applications for pelvic floor exercise on urinary incontinence. These studies and their findings are summarized below:

In a randomized controlled study conducted by Arauja et al. (2019), the effects of mobile application prepared to ensure patients' continuity to pelvic floor exercise were evaluated on urinary symptoms. 33 women (intervention group: 17, control group: 16) diagnosed with UI were included in the study. The same pelvic floor muscle exercise program was applied to both intervention and control groups. The exercise program applied, consisted of 8 seconds retention / 8 seconds relaxation, 3 times in a row, repeated 8 times. This application was repeated 2 times a day for 3 months. These exercises were applied to the initiative group via mobile application. In this mobile application, an electromyography image is provided to match the application of the pelvic floor exercise without a vaginal probe and it has a reminder that reminds the exercise application twice a day. When the intervention group heard the ringtone, which is the reminder of the application, they made the exercise program. The control group was given printed material and asked to apply the same exercise protocol twice a day at any time of the day. The results were evaluated using pelvic floor muscle examination, The International Consultation on Incontinence Questionnaire: ICIQ, electromyography. The women were examined again at the first appointment and then in the 1st, 2nd and 3rd months. As a result of this study; It has been concluded that this application, which includes pelvic floor exercise, increases the compliance of patients to home exercise program compared to written material. At the same time, it was found that women using the application exercised more than those who received written material and decreased incontinence symptoms (Araujo et al., 2019).

The effect of mobile application in the treatment of women with stress urinary incontinence was evaluated in a randomized controlled study conducted by Asklund et al. (2016). 123 women diagnosed with stress urinary incontinence (intervention group: 62, control group: 61) were participated in the study. Women in the intervention group used the mobile application Tät $\AA 2$ times a day for 3 months, which includes information about pelvic floor training, stress urinary incontinence and lifestyle advice prepared by experts. Women in the control group did not receive any intervention and their treatment was delayed for 3 months. The International Incontinence Questionnaire (Urinary Incontinence Short Form: ICIQ-UI SF) was used to evaluate the urinary system symptoms, and the Urinary System Symptoms Quality of Life Questionnaire (ICIQ: Lower Urinary Tract Symptoms Quality of Life) was used to evaluate the situation-specific quality of life. Compared to women in the control group, it was concluded that women in the intervention group had fewer symptoms, improved quality of life, healed faster, had less frequent weekly urinary incontinence, and used less pads (Asklund et al., 2016).

In the randomized controlled study conducted by Hoffman et al. (2017) as a continuation of the study of Asklund et al. (2016), the long-term effects of the 2-year use of mobile application for pelvic floor exercise in women with stress urinary incontinence were 
evaluated. In the previous study, 46 of the 62 women in the intervention group continued to use the same practice for two years. 61 women were included in the control group. The exercise program, which is included in the mobile application (Tät $\left.{ }^{\circledR}\right)$, consists of 8 seconds of retention / 8 seconds of relaxation, 3 times in a row, repeated 8 times. This practice was repeated 3 times a day for 2 years. The research was completed with 46 women in the intervention group and 60 women in the control group. The International Incontinence Questionnaire (Urinary Incontinence Short Form: ICIQ-UI SF) was used to evaluate the results and the Lower Urinary Tract Symptoms Quality of Life (ICIQ: Lower Urinary Tract Symptoms Quality of Life) was used to evaluate the situation-specific quality of life. This mobile application, which was prepared for the treatment of stress urinary incontinence at the end of this study, was observed to decrease the severity of symptoms and increase the quality of life at 2-year follow-up (Hoffman, Söderström, \& Samuelsson, 2017).

Other studies for mobile applications involving pelvic floor exercise in recent years are summarized below:

Latorre et al. (2018) conducted a systematic review to figure out mobile applications and introduce a mobile application called iPelvis. In this systematic review study, Pubmed, Embase, CINAHL, LILACS, PEDro, and Scielo search engines were scanned. Of the applications, prepared for pelvic floor training, 12 variables were determined to create the most suitable mobile application. In the examination, 61 Android and 16 Apple mobile applications were examined and it was found that none of these applications had 12 variables. iPelvis application is prepared according to these 12 variables. The examined variables; identification of the relevant bladder and pelvic problem, micturition diary, case-specific questionnaires, pelvic floor training protocol, educational steps arranged according to the patient's needs, a funny character that stimulates the patient's positive attitude considering the age, condition and ethnic group, recommended, adequate and effective behavioural therapy protocol (eg. recommendations regarding drinking), empathic verbal and visual instructions for optimum use of pelvic floor exercises, especially for the early stages of pelvic floor exercise; an effective feedback protocol to instruct the patient to find and feel the contraction and relaxation of the pelvic floor muscle remind the patient through notifications constantly to continue and encourage the application. As a result of this review study, although there are many mobile applications in pelvic floor exercise, it was emphasized that no application is evidence-based and has deficiencies. As the developed iPelvis application contains a evidence-based approach, it has been concluded that it will provide ease of access and care as a primary care service (Latorre et al., 2019).

Nyström et al. (2017) examined the factors associated with the success of the mobile application Tät ${ }^{\circledR}$ for pelvic floor exercise used in the treatment of stress incontinence. This study was conducted with 61 women who experienced stress incontinence problems at least once a week and who used the mobile application called Tät ${ }^{\circledR}$ for 3 months. In addition to being the main theme of pelvic floor exercise, Tät ${ }^{\circledR}$ 's mobile application contains information about stress incontinence, lifestyle advice, a reminder that reminds them to do a pelvic floor exercise and it has statistical usage rates of the individual. In this study, 56\% of women thought that the mobile application was successful in treatment. It has been emphasized that providing weight control, increasing the commitment to treatment and gaining selfmanagement to patients play an important role in the success of the application (Nyström et al., 2017).

In the qualitative study of Asklund et al. (2019); the experiences of women using mobile applications for stress urinary incontinence were investigated. This qualitative study was 
conducted with 15 women selected among women using mobile applications in the study of Asklund et al. (2016). Interviews were made with these women by telephone conversations using a semi-structured interview guide. As a result of this study, women using mobile applications reported that the mobile application provided their management in treatment. At the same time, women stated that implementation is a useful, easily accessible, time-saving, inexpensive, new and modern tool. On the other hand, some women stated that they experienced insecurity about whether they were practicing correctly (Asklund, Samuelsson, Hamberg, Umefjord, \& Sjöström, 2019).

\section{Conclusion}

In conclusion, evidence-based studies in recent years have shown that mobile applications for urinary incontinence reduce symptoms of incontinence, improve quality of life, heal faster recovery, decrease the frequency of weekly urinary incontinence and are effective in maintaining pelvic floor exercise. These results show that the use of mobile applications for kegel exercise can be recommended to patients with urinary incontinence. Continuing treatment is an important issue in pelvic floor exercise and mobile applications can play an active role in the developing technology world in terms of being effective in reducing therapy as well as reducing symptoms. Further evidence-based studies are needed for the effectiveness of mobile applications. 


\section{REFERENCES}

Araujo, C., Marques, A., \& Juliato, C. (2019). The adherence of home pelvic floor muscles training using a mobile device application for women with urinary incontinence: A randomized controlled trial. Female Pelvic Medicine \& Reconstructive Surgery, 1-7. https://doi.org/10.1097/SPV.0000000000000670

Asklund, I., Nyström, E., Sjöström, M., Umefjord, G., Stenlund, H., \& Samuelsson, E. (2016). Mobile app for treatment of stress urinary incontinence: A randomized controlled trial. Neurourology and Urodynamics, 9999, 1-8. https://doi.org/10.1002/nau.23116

Asklund, I., Samuelsson, E., Hamberg, K., Umefjord, G., \& Sjöström, M. (2019). User experience of an app-based treatment for stress urinary incontinence: Qualitative interview study. Journal of Medical Internet Research, 21(3), 1-11. https://doi.org/10.2196/11296

Bo, K., Frawley, H. C., Haylen, B. T., Abramov, Y., Almeida, F. G., Berghmans, B., ... Wells, A. (2017). An International Urogynecological Association (IUGA)/International Continence Society (ICS) joint report on the terminology for the conservative and nonpharmacological management of female pelvic floor dysfunction. Neurourol Urodynam, 36, 221-244. https://doi.org/10.1002/nau.23107

Borello-France, D., Burgio, K. L., Goode, P. S., Markland, A. D., Kenton, K., Balasubramanyam, A., \& Stoddard, A. M. (2010). Adherence to behavioral interventions for urge incontinence when combined with drug therapy: Adherence rates, barriers, and predictors. Physical Therapy, 90(10), 1493-1505. https://doi.org/10.2522/ptj.20080387

Çayan, S., Yaman, Ö., Orhan, İ., Usta, M., Başar, M., Resim, S., ... Kadığ lu, A. (2016). Prevalence of sexual dysfunction and urinary incontinence and associated risk factors in Turkish women. European Journal of Obstetrics \& Gynecology and Reproductive Biology, 303-308. https://doi.org/10.1016/j.ejogrb.2016.06.030

Hamine, S., Gerth-guyette, E., Faulx, D., Green, B. B., \& Sarah, A. (2015). Impact of mHealth chronic disease management on treatment adherence and patient outcomes: A systematic review. Journal of Medical Internet Research, 17(2), 1-15. https://doi.org/10.2196/jmir.3951

Han, M. N., Grisales, T., \& Sridhar, A. (2017). Evaluation of a mobile application for pelvic floor exercises. Telemedicine and E-Health, 1-5. https://doi.org/10.1089/tmj.2017.0316

Hoffman, V., Söderström, L., \& Samuelsson, E. (2017). Self-management of stress urinary incontinence via a mobile app: Two-year follow-up of a randomized controlled trial. Acta Obstetricia et Gynecologica Scandinavica, 96(10), 1180-1187. https://doi.org/10.1111/aogs.13192

Latorre, G. F. S., de Fraga, R., Seleme, M. R., Mueller, C. V., \& Berghmans, B. (2019). An ideal e-health system for pelvic floor muscle training adherence: Systematic review. Neurourology and Urodynamics, 38, 63-80. https://doi.org/10.1002/nau.23835

Leroy, L. da S., Lúcio, A., \& Lopes, M. H. B. de M. (2016). Risk factors for postpartum urinary incontinence. Journal of School of Nursing, 50(2), 200-207.

Loohuis, A. M. M., Wessels, N. J., Jellema, P., Vermeulen, K. M., Hove, M. C. S., van Gemert-Pijnen, J. E. W. C., ... Blanker, M. H. (2018). The impact of a mobile applicationbased treatment for urinary incontinence in adult women: Design of a mixed-methods randomized controlled trial in a primary care setting. Neurourology and Urodynamics, 37, 2167-2176. https://doi.org/10.1002/nau.23507 
Milsom, I., \& Gyhagen, M. (2019). The prevalence of urinary incontinence. Climacteric, 22(3), 217-222. https://doi.org/10.1080/13697137.2018.1543263

Nyström, E., Asklund, I., Sjöström, M., Stenlund, H., \& Samuelsson, E. (2017). Treatment of stress urinary incontinence with a mobile app: Factors associated with success. International Urogynecology Journal, 29(9), 1325-1333. https://doi.org/10.1007/s00192-017-3514-1

Radzimińska, A., Strączyńska, A., Weber-Rajek, M., Styczyńska, H., Strojek, K., \& Piekorz, Z. (2018). The impact of pelvic floor muscle training on the quality of life of women with urinary incontinence: a systematic literature review. Clinical Interventions in Aging, 13, 957965.

Saboia, D. M., Vasconcelos, C. T. M., Oriá, M. O. B., Bezerra, K. D. C., Ananias, J., Neto, V., \& Lopes, M. H. B. de M. (2019). Continence app: Construction and validation of a mobile application for postnatal urinary incontinence prevention. European Journal of Obstetrics \& Gynecology and Reproductive Biology, 240, 330-335. https://doi.org/10.1016/j.ejogrb.2019.07.026

"Digital 2019: Global internet use accelerates". Access Address: https://wearesocial.com/blog/2019/01/digital-2019-global-internet-use-accelerate (Access November 13, 2019) 